REVIEW

\title{
Risk of spontaneous miscarriage in euthyroid women with thyroid autoimmunity undergoing IVF: a meta-analysis
}

\author{
Konstantinos A Toulis ${ }^{1}$, Dimitrios G Goulis ${ }^{1}$, Christos A Venetis ${ }^{2}$, Efstratios M Kolibianakis ${ }^{2}$, Roberto Negro ${ }^{3}$, \\ Basil C Tarlatzis ${ }^{2}$ and Ioannis Papadimas ${ }^{1}$ \\ ${ }^{1}$ Unit of Reproductive Endocrinology and ${ }^{2}$ Unit for Human Reproduction, First Department of Obstetrics and Gynecology, Medical School, Papageorgiou \\ General Hospital, Aristotle University, Ring Road, Nea Efkarpia, 56403 Thessaloniki, Greece and ${ }^{3}$ Department of Endocrinology, Azienda Ospedaliera, \\ 'V. Fazzi', Piazza F. Muratore, 73100 Lecce, Italy \\ (Correspondence should be addressed to K A Toulis; Email: toulis@endo.gr)
}

\begin{abstract}
Objective: To investigate whether thyroid autoimmunity (TAI) is associated with increased risk for spontaneous miscarriage in subfertile, euthyroid women undergoing IVF.

Design: Meta-analysis of observational studies.

Patient(s): Four prospective studies that reported data on 1098 subfertile women undergoing IVF (141 with TAI and 957 controls) were included in the meta-analysis.

Main outcome measure: Miscarriage risk ratio (RR).

Secondary outcome measures: Clinical pregnancy rate and delivery rate.

Result(s): Euthyroid, subfertile women with TAI undergoing IVF demonstrated significantly higher risk for miscarriage compared with controls (four studies-fixed effects RR: 1.99, 95\% confidence interval: $1.42-2.79, P<0.001)$. No significant difference in clinical pregnancy and delivery rates was detected between groups.

Conclusion: Based on the currently available evidence, it appears that the presence of TAI is associated with an increased risk for spontaneous miscarriage in subfertile women achieving a pregnancy through an IVF procedure.
\end{abstract}

European Journal of Endocrinology 162 643-652

\section{Introduction}

The association between spontaneous miscarriage and thyroid autoimmunity (TAI) defined as the presence of autoantibodies against thyroid peroxidase (TPOab) and/or thyroglobulin (TGab) was initially reported by Stagnaro-Green et al. (1), subsequently investigated in numerous studies and finally confirmed by two metaanalyses $(2,3)$. Causality remains unclear; co-presence of TAI with other autoimmune syndromes, direct action of anti-TPO and TGab on placenta, hampered adaptability of the thyroid gland to the increased demands of pregnancy in the presence of TAI and higher age of women with TAI have all been implicated. The obscure pathophysiology is probably one of the reasons why adequate treatment strategies have not yet been developed. A well-designed randomized controlled trial, in which sodium levothyroxine $\left(\mathrm{L}-\mathrm{T}_{4}\right)$ supplementation was associated with a risk of miscarriage similar to that observed in healthy controls (4), needs to be confirmed before $\mathrm{L}_{-} \mathrm{T}_{4}$ supplementation could be routinely recommended in the course of a TAI-positive pregnancy (5).
Although devastating on a personal level, pregnancy loss could be regarded as a natural outcome of an inefficient process, considering that only $\sim 23 \%$ of conceptions result in live births (6). However, pregnancy outcome becomes a matter of copious and costly efforts in subfertile women undergoing artificial reproduction techniques (ART), where any factor that could potentially affect the outcome should be adequately analyzed and carefully considered. Currently, it is not clear whether there is an association between TAI and poor IVF outcome $(5,7)$. Several studies which examined the association between TAI status and miscarriage provided rather controversial results (8-18), although it appears that 'TAI per se does not alter the implantation of embryo' (19). Moreover, observations regarding TAI and miscarriage risk from the general population could not be applied to an IVF setting and vice versa, since women undergoing IVF are considered as a distinct subgroup, representing a 'special population' (20). An answer to whether the presence of thyroid autoantibodies is associated with an increased risk for miscarriage in IVF might assist the IVF counseling in women with TAI. For that purpose, 
a meta-analysis of observational studies evaluating this research question was performed.

In short, the ideal study should be prospective in design, include only first-time ART users and analyze only first cycle ART outcomes, measure both TPOab and TGab in all the subjects before ART procedure, control for the possible confounding effect of other autoantibodies and/or causes of spontaneous miscarriages and involve euthyroid, without TAI, age-matched women undergoing IVF as controls.

\section{Methods}

\section{Search strategy}

A preliminary search was conducted in the electronic database MEDLINE on various combinations of the terms 'thyroid gland' (MeSH), 'thyroid microsomal antibodies' (substance name), 'anti-thyroglobulin' (substance name), 'autoantibodies' (MeSH), 'thyroiditis, autoimmune' (MeSH), 'thyroid diseases', 'IVF' (MeSH) and 'reproductive techniques, assisted' (MeSH) in order to evaluate the size of the relevant literature and orientate to the keywords which would be used in the main search. To identify eligible studies, the main search was conducted in the electronic databases MEDLINE and EMBASE from inception through to June 2009 and was restricted to English literature. The terms used for the MEDLINE search were ('autoantibodies' (MeSH) and 'thyroid gland' (MeSH)) or 'thyroid diseases' (MeSH)) and 'reproductive techniques, assisted' (MeSH). The procedure was concluded by the perusal of the reference sections of all relevant studies or reviews and a manual search of key journals and abstracts from the major annual meetings in the field of reproduction. The main search, as well as screening of titles, abstracts, and fulltext articles, was completed independently by two reviewers (K T and D G). Any discrepancy was solved unanimously by discussion.

\section{Eligibility of relevant studies}

Studies eligible for the systematic review were those that reported data on IVF outcome in subfertile women whose TAI status had been determined, independently of design (prospective or retrospective, IVF protocol). Exclusion criteria were i) recurrent miscarriages during IVF, ii) subfertile women with known autoimmune disease other than TAI, iii) no control group or control group other than women without TAI who underwent IVF, iv) ovulation induction without IVF and v) reviews or letters to the editors.

To ensure interpretable data synthesis, a study was included into the meta-analysis, only if i) it was prospective in design, ii) euthyroid women had been enrolled, iii) TAI and control groups were comparable in age and iv) follow-up period lasted at least until the end of the first trimester of pregnancy. Studies in which it was clearly stated that analysis included multiple-cycle IVF outcomes were not considered eligible for the meta-analysis.

\section{Data extraction}

Information from each study was extracted independently by two reviewers ( $\mathrm{K}$ T and $\mathrm{D} G$ ), using a standardized data extraction form. General characteristics of the study (author, year of publication, country, study design, study period, sample size), characteristics of the study groups, their comparability on baseline characteristics (age, body mass index, thyroid function tests), methodology (IVF protocol, number of oocytes retrieved and embryos transferred, biochemical and clinical pregnancy definition, thyroid autoantibodies measurement method, threshold and time of measurement, study quality), and outcomes (biochemical and clinical pregnancies, deliveries, miscarriages) were recorded, where available, and double-checked. Where appropriate, an effort was made to complete the data set through communication with the authors.

\section{Outcomes}

The main outcome of the meta-analysis was the miscarriage risk, which was defined as the loss of

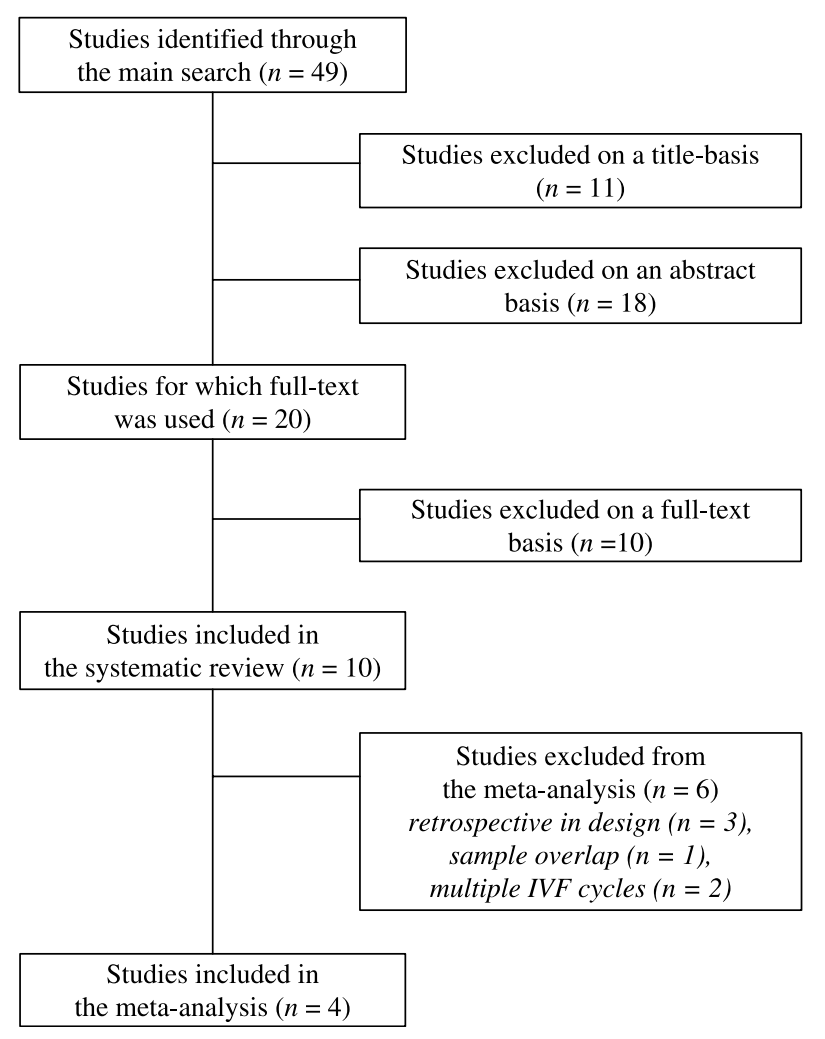

Figure 1 Flow chart for the systematic review and the metaanalysis. 


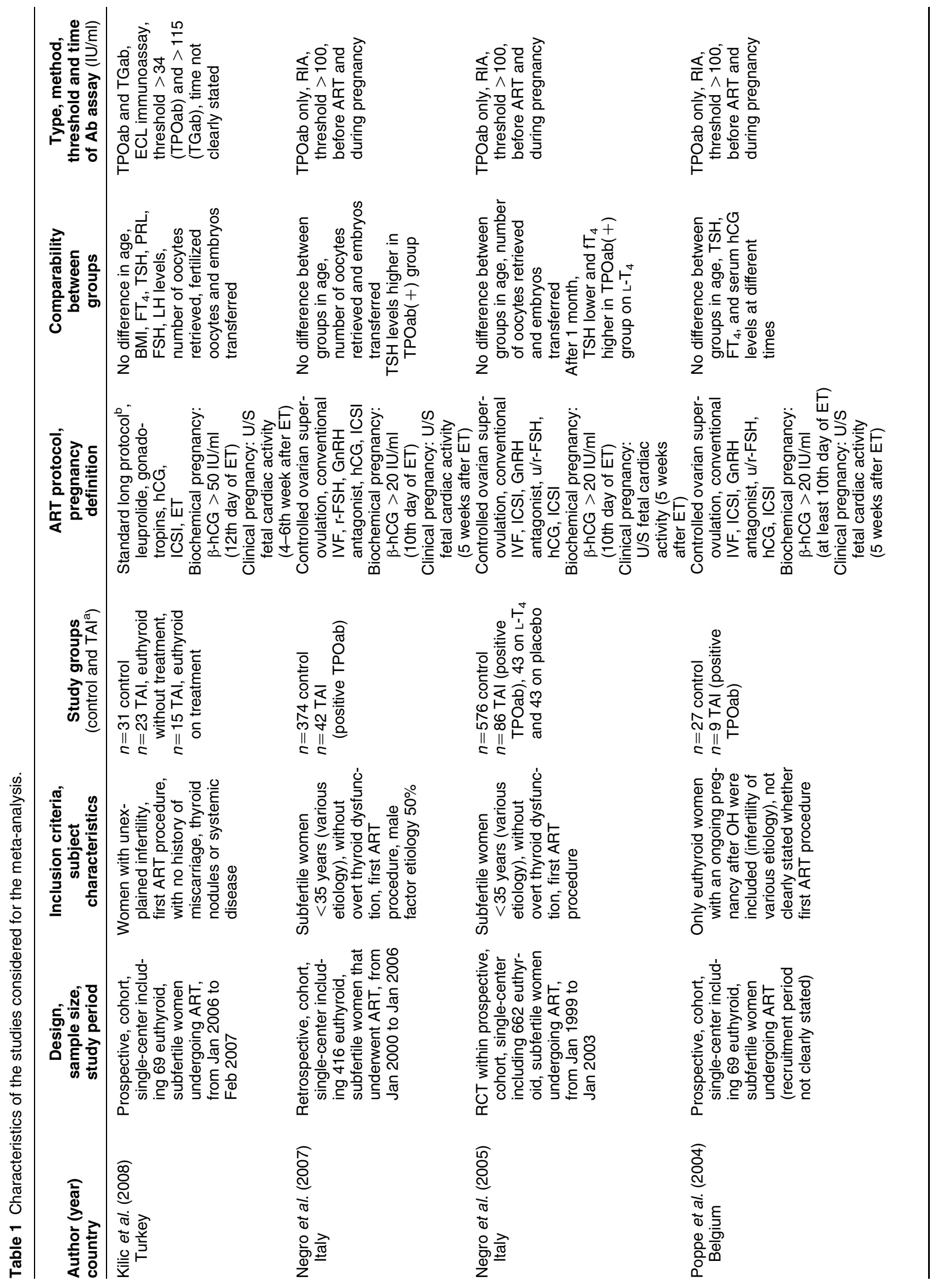




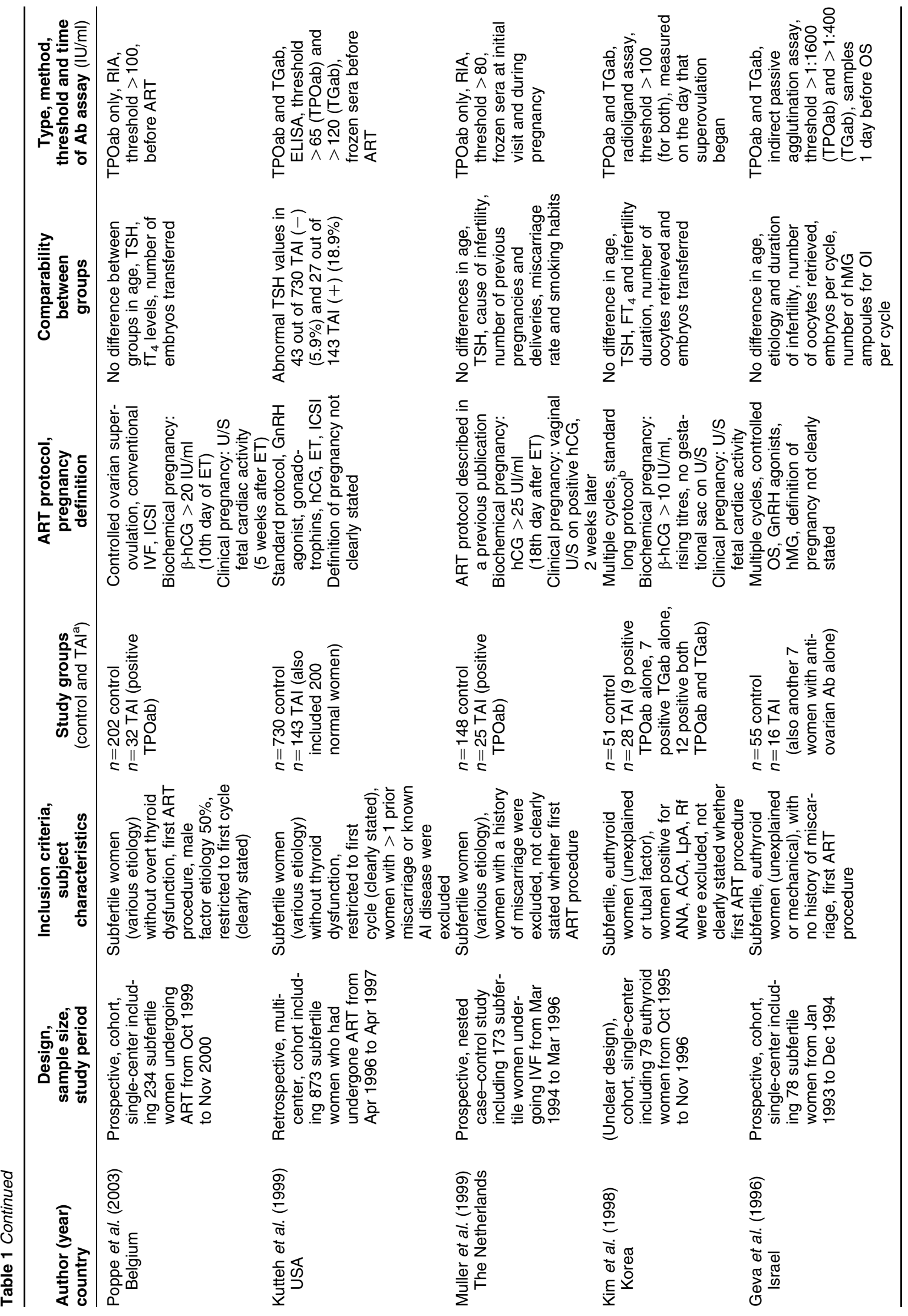

www.eje-online.org 


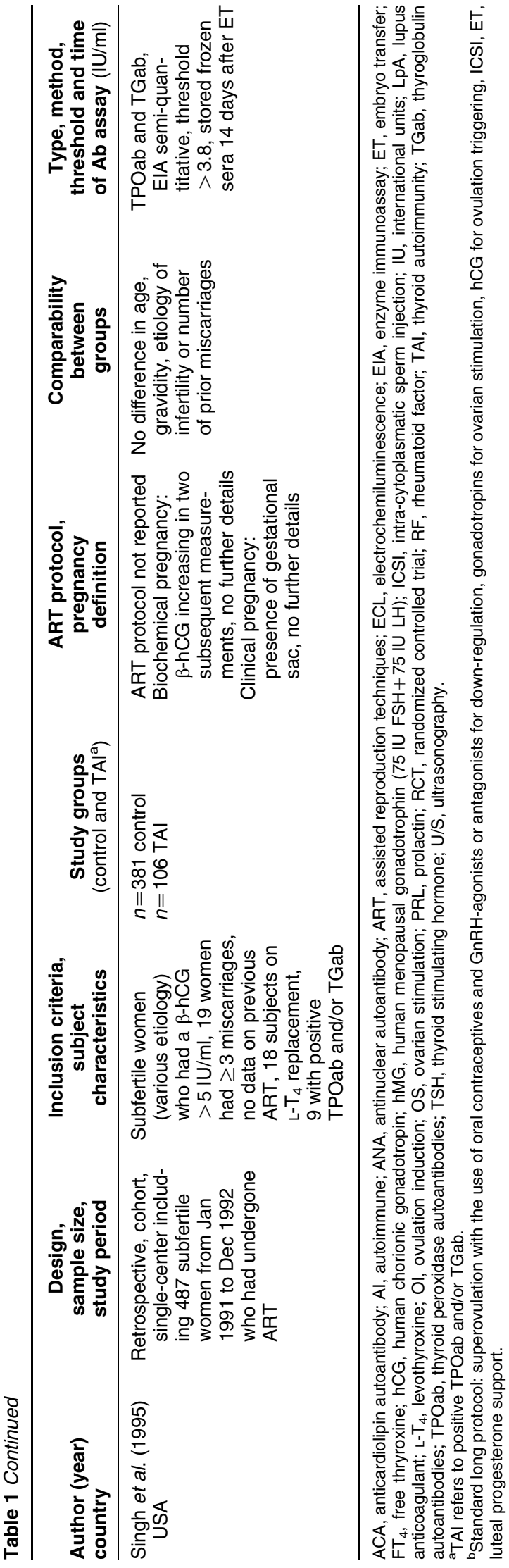

pregnancy after a positive confirmation, either biochemical or clinical. In order to gain a more thorough perception of IVF outcome in women with TAI, clinical pregnancies (ultrasound confirmed fetal cardiac activity, 5 weeks after embryo transfer (ET)) and deliveries served as secondary outcome measures.

\section{Statistical analysis}

As a dichotomous outcome, miscarriage risk in each study was expressed as risk ratio (RR) with 95\% confidence interval (CI) and combined using a fixed effects model and Mantel-Haensel method as the weighting scheme. When significant heterogeneity was detected, random effects model was preferred. Secondary outcomes were analyzed similarly.

Heterogeneity between the results of different studies was examined by $I^{2}$ test $\left(I^{2}>50 \%\right.$ : significant heterogeneity, $I^{2}=50-25 \%$ : moderate heterogeneity, $I^{2}<25 \%$ : insignificant heterogeneity), which can be interpreted as the percentage of total variation across several studies due to heterogeneity (21). To assess the extent of publication bias, Egger's test for publication bias was used (22). Sensitivity analyses were undertaken with the exclusion of a study with borderline eligibility. Meta-analysis was conducted using Stata/SE 10.0 for Windows (StataCorp. LP, 4905 College Station, TX, USA). The report of the study was complemented in adherence with the meta-analysis of observational studies in epidemiology group standards for reporting meta-analysis of observational studies (23).

\section{Results}

\section{Search results}

The search strategy identified 49 potentially relevant studies. Seven of them were identified through reference sections of relevant publications or manual search. A flow chart summarizing search results is provided in Fig. 1. Eleven publications were excluded, because it was clear from the title that they did not fulfill the selection criteria. From the remaining 38 publications, 18 were excluded on the basis of the abstract. Twenty articles were read in full, independently by two reviewers, to assess their accordance with the predefined inclusion criteria. Four studies were excluded because TAI status had not been determined (24-26) or women with TAI had been excluded (27). Three studies were excluded because women with recurrent spontaneous miscarriages had been recruited (28-30) and another three studies because the IVF outcome had not been recorded or presented $(14,31,32)$.

From the remaining ten studies included in the systematic review, three were excluded from the metaanalysis on the basis of their retrospective design (15-17), two because women involved underwent multiple IVF procedures $(8,9)$, and one (18) because 


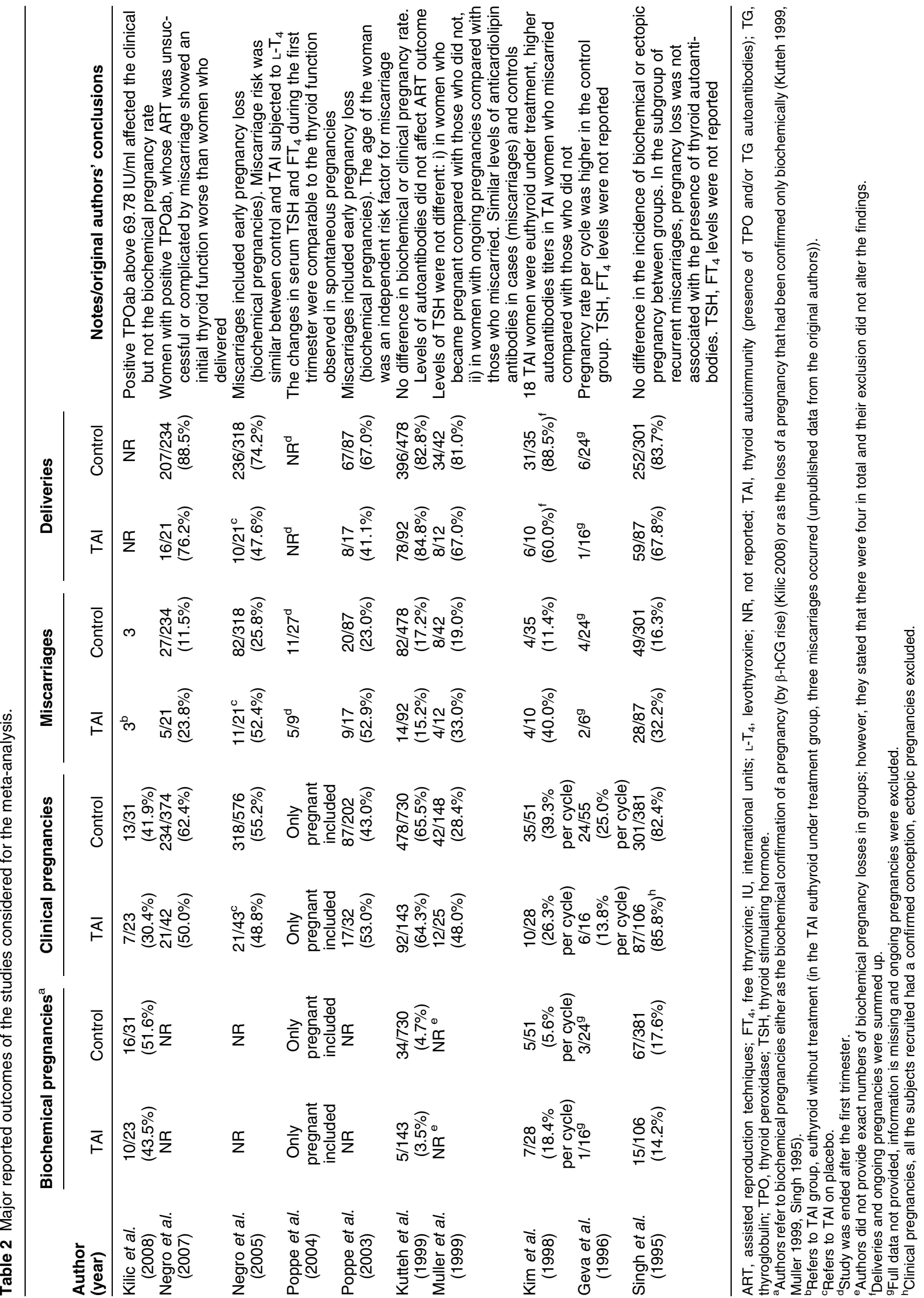


of a sample overlap with a previous study (12). Finally, four studies were included in the meta-analysis (10-13, 18). Disagreement between reviewers was recorded on whether multiple IVF procedures were involved in some of the included studies $(10,11,13,18)$ and was resolved by consensus.

\section{Systematic review}

The ten studies included in the systematic review were published between 1995 and 2008 and reported data on 3107 subfertile women undergoing IVF procedures. Main data are summarized in Tables 1 and 2. Five of them were held in Europe, three in Asia, and two in the United States. Substantial diversity was observed in the methodology researchers had chosen to investigate the hypothesis. Six studies were prospective in design, three were retrospective, while in one study, the design was not clearly stated (8). In five studies, it was clearly stated that first ART procedure was an inclusion criterion, whereas in three studies, no specific statement was provided $(8,13,15,18)$. In two studies, it was clearly reported that only first cycle results were analyzed $(12,15)$, in another two, multiple IVF cycles were reported $(8,9)$, whereas in the rest no relevant information could be retrieved. Groups were comparable in age in all of the studies but differed in mean thyroid stimulating hormone (TSH) values in two studies $(15,17)$. TSH and free $\mathrm{T}_{4}\left(\mathrm{FT}_{4}\right)$ levels were not reported in two studies $(9,16)$.

Diversity in the methodology applied to measure thyroid autoantibodies was observed too. Both TPOab and TGab were determined in five studies, whereas in the remaining five, only TPOab was measured (11-13, 17, 18). Most studies (generally the most recent) applied RIA for the measurements (five studies), whereas electrochemiluminescence assays (ECL), enzyme immunoassays, ELISA, indirect passive agglutination, and radioligand assays were also used with various thresholds (further details in Table 1). In one study, sera were drawn 14 days after ET (16), and in another, no relevant information was provided (10).

Overall, the reporting of studies was fair: total numbers, inclusion criteria, baseline characteristics, and IVF protocols were generally clearly stated; however, reporting on secondary outcomes (gestational age when miscarriage occurred, biochemical pregnancies, levels of antibodies) and on limitations was incomplete.

\section{Meta-analysis}

The four studies that were included in the meta-analysis reported data on 1098 subfertile women undergoing IVF procedures (141 with TAI and 957 controls). Study size varied from 69 to 622 women (median=203.5). The percentage of miscarriage (to clinical pregnancies) reported in individual studies ranged from 33.3 to $52.9 \%$. Main results of the meta-analysis are summarized below.

\section{Miscarriage risk}

Subfertile women with TAI undergoing IVF demonstrated significantly higher risk for miscarriage compared with subfertile women without TAI undergoing IVF (four studies-fixed effects RR: 1.99, 95\% CI: 1.416-2.793, $P<0.001$; Fig. 2). No sign of heterogeneity among studies was detected $\left(I^{2}=0 \%\right)$. No sign of publication bias was detected (Egger's test, $P=0.298$ ). Sensitivity analysis, with the exclusion of the study by Kilic et al. revealed similar findings (three studies-fixed effects RR: 2.07, 95\% CI: 1.47-2.71).

\section{Clinical pregnancy rate}

Subfertile women with TAI undergoing IVF demonstrated similar clinical pregnancy rate compared with subfertile women without TAI undergoing IVF (four studies-random effects RR: 1.107, 95\% CI: 0.80-1.530, $P=0.504$; Fig. 3); however, substantial heterogeneity

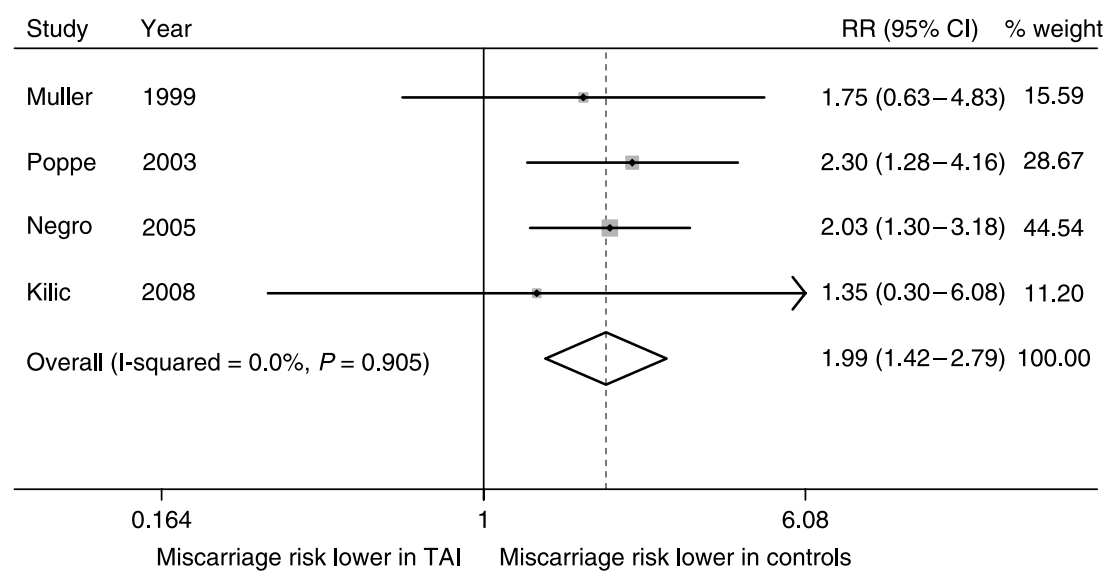

Figure 2 Miscarriage risk in euthyroid women with TAI undergoing IVF. 


\begin{tabular}{|c|c|c|c|}
\hline Study & Year & $\mathrm{RR}(95 \% \mathrm{Cl})$ & $\%$ weight \\
\hline Muller & 1999 & $1.69(1.04-2.74)$ & 23.30 \\
\hline Poppe & 2003 & $1.23(0.86-1.77)$ & 30.03 \\
\hline Negro & 2005 & $0.88(0.65-1.21)$ & 33.07 \\
\hline Kilic & 2008 & $0.73(0.34-1.53)$ & 13.60 \\
\hline Overall & $($ I-squared $=54.1 \%, P=0.088)$ & $1.11(0.80-1.53)$ & 100.00 \\
\hline \multicolumn{4}{|c|}{ NOTE: Weights are from random effects analysis } \\
\hline & 0.345 & 2.9 & \\
\hline
\end{tabular}

Figure 3 Clinical pregnancy rate in euthyroid women with TAI undergoing IVF. among studies was detected $\left(I^{2}=54.1 \%\right)$. Sensitivity analysis, with the exclusion of the study by Kilic et al. revealed similar findings (three studies-random effects RR: 1.18, 95\% CI: 0.88-1.69).

\section{Delivery rate}

Subfertile women with TAI undergoing IVF demonstrated similar delivery rate compared with subfertile women without TAI undergoing IVF (three studiesrandom effects RR: 0.826 , 95\% CI: $0.488-1.398$, $P=0.477$; Fig. 4). Substantial heterogeneity among studies was detected $\left(I^{2}=55.6 \%\right)$.

\section{Discussion}

The present meta-analysis attempted to provide an estimation of the risk for miscarriage for euthyroid women undergoing IVF in the presence of thyroid autoantibodies. Despite the relative paucity of primary data (four studies) that undermine the reliability of the results, it was estimated that a subfertile euthyroid woman with TAI is at a twofold higher risk of miscarriage in an IVF pregnancy compared with a counterpart without TAI. The result is in agreement with those of similar studies on spontaneous (non-IVF) pregnancies (3), both in the direction and in the magnitude of the effect. Furthermore, it appears to be consistent across studies, since no sign of heterogeneity was detected. On the other hand, the present metaanalysis failed to detect a significant effect of TAI status on the clinical pregnancy rate and delivery rate in women undergoing IVF. However, the latter finding should be regarded with caution, since only three studies reported delivery rates; thus, the analysis was probably underpowered. In fact, a post hoc power analysis revealed that a sample size of $\sim 300$ women with TAI would be needed to detect a difference in delivery rates (study power 0.8 , TAI:control allocation 1:9). Alternatively, it could be suggested that, despite a twofold relative increase in the risk of miscarriage, the actual difference in the number of miscarriages between euthyroid women with TAI undergoing IVF and controls (the attributable risk) is small, resulting in a rather 'silent' effect on delivery or clinical pregnancy rate.

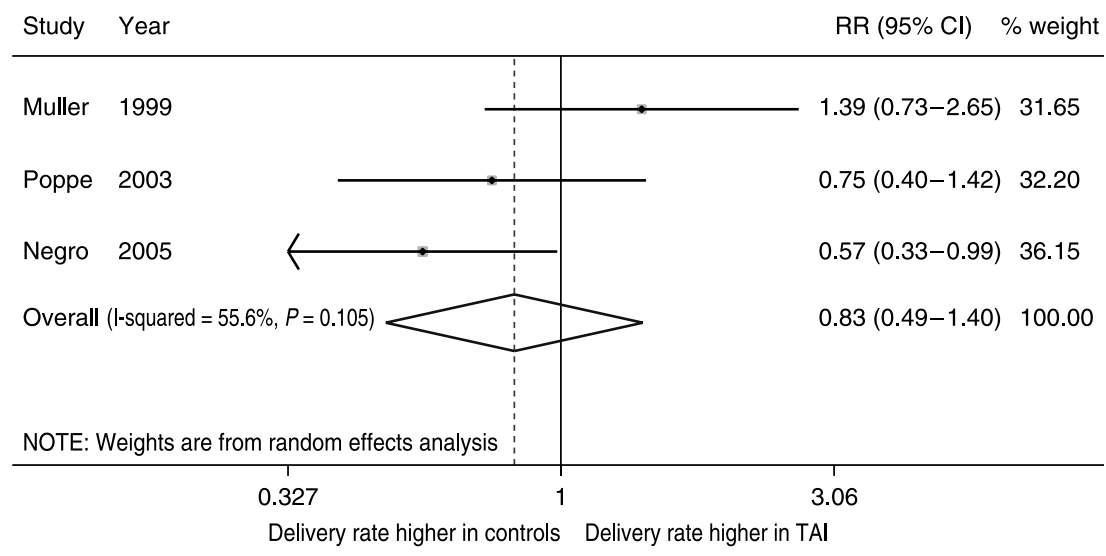

Figure 4 Delivery rate in euthyroid women with TAI undergoing IVF. 
The findings of the present meta-analysis could potentially provide useful inferences about the etiology of the association between TAI and miscarriage in IVF. First, the comparison between the TAI-related miscarriage risk in IVF pregnancies and similar risk in spontaneous pregnancies (3) is consistent with the fact that the exogenous use of gonadotropins in IVF procedures does not convey additional risk for miscarriage in the presence of TAI. Despite evidence for a pattern of dissociation for both serum TSH (higher) and $\mathrm{FT}_{4}$ (lower) in women with TAI compared with those without TAI after ART-induced pregnancies (18), the clinical importance of the changes provoked by gonadotropins in terms of miscarriage risk appears to be minimal.

On the contrary, it appears that the role of TSH levels and $\mathrm{L}-\mathrm{T}_{4}$ supplementation are important for IVF outcome. Poppe et al. investigated the thyroid function after an IVF procedure and its association with the reproductive outcome (18). Although it failed to reach statistical significance in thyroid function parameters between miscarriage and ongoing pregnancy groups, the study did confirm a significantly different pattern of change in thyroid function during the very first period of pregnancy' between TAI groups. Negro et al. provided evidence that $\mathrm{L}-\mathrm{T}_{4}$ supplementation in women with TAI who became pregnant through IVF did lower the miscarriage risk to the level of controls (11). Subsequently, the same group reported in a retrospective study that women with TAI, whose ART was unsuccessful or complicated by miscarriage, showed worse thyroid function profile than women who delivered (17). Unfortunately, although subtle alterations in thyroid function could potentially explain the observed difference in miscarriage risk in the present meta-analysis, their impact was not investigated, since thyroid function tests were not applied in the course of IVF pregnancies in most studies. The lack of an assessment of the dynamics of thyroidal function or the actual thyroid status at miscarriage, which could have favored an etiopathogenic approach, inserts bias in the analysis that can actually affect the interpretation of clinical data.

In regard to alternative explanations for the observed difference in miscarriage, the influence of age is rather minimal, since all the TAI groups were comparable in the mean value in all the studies included in the present meta-analysis. A 'dose-response' pattern between autoantibody titers and miscarriage risk, which could potentially imply a direct action, could not be supported by the findings of the systematic review; the unique report of an association between them (8) was not confirmed subsequently (15). On the other hand, influence of other causes of spontaneous abortions was not routinely controlled for; the presence of other autoantibodies was an exclusion criterion in only one study (8), whereas another one retrospectively estimated the prevalence of anticardiolipin antibodies (13).
Etiology of infertility was reported to be similar in TAI groups in four studies $(9,11,16,33)$, while three evaluated personal history of miscarriages as an exclusion criterion $(9,10,13)$. Thus, the potential confounding effect of other causes of spontaneous miscarriages could not be neglected.

Recently, another hypothesis has been suggested to explain the association between TAI and miscarriage (34), compatible with the notion of thyroid autoantibodies as a marker that should be regarded as a reflection of an undefined autoimmunity. According to this hypothesis, the presently undefined autoimmunity is the blocking TSH receptor autoantibodies (TSHRabblocking), not infrequently detected in Hashimoto's thyroiditis (35), which could putatively inhibit, through a cross-reactivity process (34), the action of the human chorionic gonadotropin (hCG) on the corpus luteum. This inhibition could lead to a decrease in progesterone and estrogen production that is essential for the support and maintenance of pregnancy during the first trimester (36). Unfortunately, neither TSHRab nor progesterone levels were reported in the studies included in the systematic review. In one study where hCG levels were measured (18), their mean value appeared to grow slower in the TAI group than in the control group, yet in a non-significant fashion.

In summary, it could be argued that euthyroid, subfertile women with TAI face an approximately twofold higher risk for miscarriage when undergoing an IVF compared with subfertile women without TAI. However, due to the relative paucity of evidence, absence of an established intervention and cost, the routine screening of thyroid autoantibodies before an IVF procedure should be reserved in a research setting.

\section{Declaration of interest}

The authors declare that there is no conflict of interest that could be perceived as prejudicing the impartiality of the research reported.

\section{Funding}

This research did not receive any specific grant from any funding agency in the public, commercial, or not-for-profit sector.

\section{Acknowledgements}

We are very grateful to Dr Kris Poppe (Vrije Universiteit, Brussels, Belgium) and Dr Sevtap Kilic (Women's Health and Research Hospital, Ankara, Turkey) who both provided additional data on their publications.

\section{References}

1 Stagnaro-Green A, Roman SH, Cobin RH, el-Harazy E, AlvarezMarfany M \& Davies TF. Detection of at-risk pregnancy by means of highly sensitive assays for thyroid autoantibodies. Journal of the American Medical Association 1990264 1422-1425. 
2 Poppe K \& Glinoer D. Thyroid autoimmunity and hypothyroidism before and during pregnancy. Human Reproduction Update 20039 149-161.

3 Prummel MF \& Wiersinga WM. Thyroid autoimmunity and miscarriage. European Journal of Endocrinology 2004150 751-755.

4 Negro R, Formoso G, Mangieri T, Pezzarossa A, Dazzi D \& Hassan H. Levothyroxine treatment in euthyroid pregnant women with autoimmune thyroid disease: effects on obstetrical complications. Journal of Clinical Endocrinology and Metabolism 200691 2587-2591.

5 Abalovich M, Amino N, Barbour LA, Cobin RH, De Groot LJ, Glinoer D, Mandel SJ \& Stagnaro-Green A. Management of thyroid dysfunction during pregnancy and postpartum: an Endocrine Society Clinical Practice Guideline. Journal of Clinical Endocrinology and Metabolism 200792 S1-S47.

6 Zinaman MJ, Clegg ED, Brown CC, O'Connor J \& Selevan SG. Estimates of human fertility and pregnancy loss. Fertility and Sterility $1996 \mathbf{6 5} 503-509$.

7 Stagnaro-Green A \& Glinoer D. Thyroid autoimmunity and the risk of miscarriage. Best Practice \& Research. Clinical Endocrinology $\mathcal{E}$ Metabolism 200418 167-181.

$8 \mathrm{Kim} \mathrm{CH}$, Chae HD, Kang BM \& Chang YS. Influence of antithyroid antibodies in euthyroid women on in vitro fertilization-embryo transfer outcome. American Journal of Reproductive Immunology $1998402-8$.

9 Geva E, Vardinon N, Lessing JB, Lerner-Geva L, Azem F, Yovel I, Burke M, Yust I, Grunfeld R \& Amit A. Organ-specific autoantibodies are possible markers for reproductive failure: a prospective study in an in-vitro fertilization-embryo transfer programme. Human Reproduction 199611 1627-1631.

10 Kilic S, Tasdemir N, Yilmaz N, Yuksel B, Gul A \& Batioglu S. The effect of anti-thyroid antibodies on endometrial volume, embryo grade and IVF outcome. Gynecological Endocrinology 2008 24 649-655.

11 Negro R, Mangieri T, Coppola L, Presicce G, Casavola EC, Gismondi R, Locorotondo G, Caroli P, Pezzarossa A, Dazzi D \& Hassan H. Levothyroxine treatment in thyroid peroxidase antibody-positive women undergoing assisted reproduction technologies: a prospective study. Human Reproduction 200520 1529-1533.

12 Poppe K, Glinoer D, Tournaye H, Devroey P, van Steirteghem A, Kaufman L \& Velkeniers B. Assisted reproduction and thyroid autoimmunity: an unfortunate combination? Journal of Clinical Endocrinology and Metabolism 200388 4149-4152.

13 Muller AF, Verhoeff A, Mantel MJ \& Berghout A. Thyroid autoimmunity and abortion: a prospective study in women undergoing in vitro fertilization. Fertility and Sterility 199971 30-34.

14 Kutteh WH, Yetman DL, Carr AC, Beck LA \& Scott RT Jr. Increased prevalence of antithyroid antibodies identified in women with recurrent pregnancy loss but not in women undergoing assisted reproduction. Fertility and Sterility 199971 843-848.

15 Kutteh WH, Schoolcraft WB \& Scott RT Jr. Antithyroid antibodies do not affect pregnancy outcome in women undergoing assisted reproduction. Human Reproduction 199914 2886-2890.

16 Singh A, Dantas ZN, Stone SC \& Asch RH. Presence of thyroid antibodies in early reproductive failure: biochemical versus clinical pregnancies. Fertility and Sterility 199563 277-281.

17 Negro R, Formoso G, Coppola L, Presicce G, Mangieri T, Pezzarossa A \& Dazzi D. Euthyroid women with autoimmune disease undergoing assisted reproduction technologies: the role of autoimmunity and thyroid function. Journal of Endocrinological Investigation $2007 \mathbf{3 0} 3-8$.

18 Poppe K, Glinoer D, Tournaye H, Schiettecatte J, Devroey P, van Steirteghem A, Haentjens P \& Velkeniers B. Impact of ovarian hyperstimulation on thyroid function in women with and without thyroid autoimmunity. Journal of Clinical Endocrinology and Metabolism 200489 3808-3812.
19 Poppe K, Velkeniers B \& Glinoer D. Thyroid disease and female reproduction. Clinical Endocrinology 200766 309-321.

20 Glinoer D. Thyroid autoimmunity and spontaneous abortion. Fertility and Sterility $1999 \mathbf{7 2} 373-374$.

21 Higgins JP, Thompson SG, Deeks JJ \& Altman DG. Measuring inconsistency in meta-analyses. BMJ $2003 \mathbf{3 2 7} 557-560$.

22 Davey Smith G, Egger M \& Phillips AN. Meta-analysis. Beyond the grand mean? BMJ 1997315 1610-1614.

23 Stroup DF, Berlin JA, Morton SC, Olkin I, Williamson GD, Rennie D, Moher D, Becker BJ, Sipe TA \& Thacker SB. Meta-analysis of observational studies in epidemiology: a proposal for reporting, meta-analysis of observational studies in epidemiology (MOOSE) group. Journal of the American Medical Association $2000 \mathbf{2 8 3}$ 2008-2012.

24 Cramer DW, Sluss PM, Powers RD, McShane P, Ginsburgs ES, Hornstein MD, Vitonis AF \& Barbieri RL. Serum prolactin and TSH in an in vitro fertilization population: is there a link between fertilization and thyroid function? Journal of Assisted Reproduction and Genetics 200320 210-215.

25 Zollner U, Lanig K, Steck T \& Dietl J. Assessment of endocrine status in patients undergoing in-vitro fertilization treatment. Is it necessary? Archives of Gynecology and Obstetrics 2001265 16-20.

26 Arojoki M, Jokimaa V, Juuti A, Koskinen P, Irjala K \& Anttila L. Hypothyroidism among infertile women in Finland. Gynecological Endocrinology $2000 \mathbf{1 4} 127-131$.

27 Poppe K, Glinoer D, Tournaye H, Schiettecatte J, Haentjens P \& Velkeniers B. Thyroid function after assisted reproductive technology in women free of thyroid disease. Fertility and Sterility 200583 1753-1757.

28 De Carolis C, Greco E, Guarino MD, Perricone C, Dal Lago A, Giacomelli R, Fontana L \& Perricone R. Anti-thyroid antibodies and antiphospholipid syndrome: evidence of reduced fecundity and of poor pregnancy outcome in recurrent spontaneous aborters. American Journal of Reproductive Immunology 200452 263-266.

29 Vaquero E, Lazzarin N, Caserta D, Valensise H, Baldi M, Moscarini M \& Arduini D. Diagnostic evaluation of women experiencing repeated in vitro fertilization failure. European Journal of Obstetrics, Gynecology, and Reproductive Biology 2006125 79-84.

30 Bussen S, Steck T \& Dietl J. Increased prevalence of thyroid antibodies in euthyroid women with a history of recurrent in-vitro fertilization failure. Human Reproduction 200015 545-548.

31 Haller K, Sarapik A, Talja I, Salumets A \& Uibo R. Controlled ovarian hyperstimulation changes the prevalence of serum autoantibodies in in vitro fertilization patients. American Journal of Reproductive Immunology 200656 364-370.

32 Klip H, van Leeuwen FE, Schats R \& Burger CW. Risk of benign gynaecological diseases and hormonal disorders according to responsiveness to ovarian stimulation in IVF: a follow-up study of 8714 women. Human Reproduction 2003 18 1951-1958.

33 De Geyter C, Steimann S, Muller B, Kranzlin ME \& Meier C. Pattern of thyroid function during early pregnancy in women diagnosed with subclinical hypothyroidism and treated with L-thyroxine is similar to that in euthyroid controls. Thyroid 200919 53-59.

34 Toulis KA, Goulis DG, Venetis CA, Kolibianakis EM, Tarlatzis BC \& Papadimas I. Thyroid autoimmunity and miscarriages: the corpus luteum hypothesis. Medical Hypotheses 20096 1060-1062.

35 Pearce EN, Farwell AP \& Braverman LE. Thyroiditis. New England Journal of Medicine 2003348 2646-2655.

36 Csapo AI, Pulkkinen MO, Ruttner B, Sauvage JP \& Wiest WG. The significance of the human corpus luteum in pregnancy maintenance. I. Preliminary studies. American Journal of Obstetrics and Gynecology $19721121061-1067$.

Received 17 November 2009

Accepted 30 November 2009 